\title{
PERFILES SOCIALES DE LA POBLACIÓN ADULTA MAYOR EN EL ESTADO DE MÉXICO, 2015
}

\section{SOCIAL PROFILES OF THE OLDER ADULT POPULATION IN THE STATE OF MEXICO, 2015}

\author{
Yuliana Gabriela Román Sánchez* \\ Sergio Cuauhtémoc Gaxiola Robles Linares** \\ Bernardino Jaciel Montoya Arce ${ }^{* * *}$ \\ Daniel Lozano Keymolen ${ }^{* * * *}$
}

RESUMEN

\begin{abstract}
El proceso de envejecimiento en la población de México se caracteriza por una serie de desigualdades relacionadas con factores sociales. El caso particular del Estado de México destaca pues se trata de la entidad con el mayor porcentaje de personas de 60 años o más. El objetivo de este artículo fue identificar perfiles sociales de la población de 60 años o más en el Estado de México con la finalidad de exponer la heterogeneidad de este grupo poblacional. Lo anterior se realizó con datos de la Encuesta Intercensal 2015 y por medio de la estimación del Análisis de Correspondencias Múltiples (АСM). En los resultados se identificaron cinco grupos de adultos mayores; donde el sexo, el nivel de escolaridad y la jubilación fueron variables fundamentales para identificar cada grupo.
\end{abstract}

PALABRAS CLAVE: MÉXICO * VEJEZ * PENSIÓN * DEMOGRAFÍA * ANÁLISIS SOCIOECONÓMICO

1 El artículo que se presenta es resultado de las interrogantes surgidas de un proyecto mayor denominado: "Vulnerabilidad social y política pública para los adultos mayores en el Estado de México". Proyecto registrado ante la Secretaría de Investigación de la Universidad Autónoma del Estado de México (UAEM).

* $\quad$ Centro de Investigación y Estudios Avanzados de la Población (CIEAP) de la Universidad Autónoma del Estado de México (UAEM).

madon_dl@hotmail.com

** Centro de Investigación y Estudios Avanzados de la Población (CIEAP) de la Universidad Autónoma del Estado de México (UAEM).

serobles99@gmail.com

*** Centro de Investigación y Estudios Avanzados de la Población (CIEAP) de la Universidad Autónoma del Estado de México (UAEм).

bjmontoyaa@uaemex.mx

Centro de Investigación y Estudios Avanzados de la Población (CIEAP) de la Universidad Autónoma del Estado de México (UAEM).

daniel.lozkey@gmail.com/dlozano@colmex.mx 


\section{ABSTRACT}

The process of aging in the population of Mexico is characterized by a series of inequalities related to social factors. The particular case of the State of Mexico stands out because it is the entity with the highest percentage of population with 60 years and over. The objective of this article was to identify social profiles of the population of 60 years or more in the State of Mexico in order to expose the heterogeneity of this population group. This was done with data from the Intercensal Survey 2015 and by means of the estimation of Multiple Correspondence Analysis (АСм). Five groups of older adults were identified in the results. Gender, schooling level and retirement were key variables to identify each group.

KEYWORDS: MÉXICO * OLD AGE * PENSIONS * DEMOGRAPHY * SOCIO-ECONOMIC ANALYSIS

\section{INTRODUCCIÓN}

El Estado de México es una de las 32 entidades de la República Mexicana; se compone de 125 municipios, 58 de los cuales son parte de dos grandes urbes: la Zona Metropolitana de la Ciudad de México y la Zona Metropolitana del Valle de Toluca, ambas ubicadas en el centro del país. La cercanía con la Ciudad de México, capital de la nación, permite que la entidad ostente un elevado dinamismo económico, incluso superior al de otros países ${ }^{2}$.

En términos demográficos, el Estado de México es la entidad más poblada de la República Mexicana. Hacia el año 2016, su población asciende a 17.1 millones, que representan 14\% de la población del país. Actualmente, su estructura por edad se compone de $26,1 \%$ de personas menores de 14 años, 63,9\% de individuos entre 15 y 59 años (económicamente activos), 9,2\% tienen 60 años o más. Este último grupo, denominado adultos mayores, configura el objeto de estudio de la presente investigación.

El proceso de envejecimiento demográfico que se experimenta en el Estado de México denota el aumento de la población de 60 años o

$2 \quad$ En el año 2011, el Estado de México aportó cerca de $10 \%$ al Producto Interno Bruto (PIB) nacional, solo por debajo del registrado por la Ciudad de México (INEGI, 2014). Dicho porcentaje convertido en miles de millones de dólares estadounidenses equivale a 189.41, cifra superior a la registrada por El Salvador (45.9), Honduras (33.74), Nicaragua (24.2) o países africanos como República Democrática del Congo (44.3), Níger (13.6), Yemen (88.5), entre otros (INEGI, 2016). más, tanto en términos absolutos como relativos; no obstante, este grupo de adultos mayores tiene diferentes trayectorias de vida que hacen de este un segmento heterogéneo. El interés por analizar a la población de la tercera edad surge por dos aspectos; en primer lugar, el acelerado crecimiento que ha tenido este grupo poblacional en las últimas décadas y en segundo lugar, por la diversidad de situaciones sociales que existen en la entidad mexiquense.

En este sentido, el objetivo de este artículo fue identificar perfiles sociales de la población de 60 años o más en el Estado de México a través de cuatro vertientes: sociodemográfica, laboral, familiar y de vivienda ${ }^{3}$. Lo que se pretende es conocer la asociación de estos para formar grupos poblacionales de adultos mayores con características comunes.

Para lograr este objetivo se utilizó el Análisis de Correspondencias Múltiples (Асм) que permite mostrar de manera gráfica, las diferencias sociales de los adultos mayores mexiquenses para el año 2015. Una de las ventajas de esta técnica estadística radica en que la visualización gráfica de las variables se puede expresar como las distancias sociales entre los individuos (Bourdieu, 2005).

El artículo está dividido en cinco apartados. El primero expone el cambio que ha tenido

$3 \quad$ Si bien, el término "perfil social" puede incluir diversos elementos, en este artículo se considera que las vertientes sociodemográfica, laboral, familiar $y$ de vivienda permiten identificar parte de la heterogeneidad entre los adultos mayores mexiquenses. 
el concepto de vejez a través del tiempo. En el segundo, se describe la composición por edad y sexo de la población adulta mayor en el Estado de México. El tercero plantea los indicadores, su cálculo, la fuente de información utilizada y los fundamentos del Análisis de Correspondencias Múltiples (АСм). En el cuarto se presenta el contexto sociodemográfico de los adultos mayores mexiquenses para el año 2015. En el quinto se expone la identificación de los grupos de los adultos mayores generados por el АсM. Por último, se presentan las conclusiones.

\section{RECORRIDO CONCEPTUAL DE LA VEJEZ}

En su concepción más amplia, la vejez es una etapa de la vida con características biológicas, sociales y culturales (Rodríguez, 1979). Las características físicas se reflejan en el deterioro de la piel, las enfermedades asociadas al envejecimiento, el detrimento de las capacidades funcionales y la discapacidad, entre otras (Rodríguez, 1979; Arber y Ginn, 1995).

En tanto, los aspectos sociales y culturales se relacionan con la posición que ostenta un sujeto dentro de la sociedad, consecuencia de las responsabilidades, los privilegios y las actividades que desarrolla (Arber y Ginn, 1995). De ahí que la condición de personas de la tercera edad depende del contexto social, pues el estatus del individuo se encuentra determinado por la colectividad (Polo y Martínez, 2001). Es importante señalar que una característica de la vejez en los países en desarrollo es la heterogeneidad de este grupo poblacional que, en muchos casos, se vincula con el contexto socioeconómico en el que se desarrolla esta etapa de la vida (Barrientos, 2006; Van de Poel, Reza, Speybroeck, Van Ourti y Vega, 2008).

El concepto de vejez es una construcción social, cultural e histórica cuyo significado se modifica en concordancia con el entorno, el espacio y el tiempo en el cual se analice (Rodríguez, 1979; Redondo, 1990; Polo y Martínez, 2001). Al respecto, en las sociedades primitivas contemporáneas pocos individuos llegaban a los 50 años de edad, por ello, se les atribuía protecciones sobrenaturales, cumplían con la función oral del conocimiento; además se desempeñaban como curanderos, chamanes $y$ dominaban los rituales; estas personas generaban miedo y respeto; los ancianos en pueblos primitivos eran considerados como individuos llenos de vigor y sabiduría (Polo y Martínez, 2001). Lo mismo ocurría en las comunidades sedentarias agrícolas-ganadera, en donde el consejo de ancianos era una de las instituciones más veneradas y respetadas con un importante poder en el plano consultivo; no obstante, el papel de la mujer era marginal e inferior al ostentado por los hombres ancianos.

En las sociedades antiguas (egipcia, griega, romana) la vejez se asociaba con el mundo de lo sagrado, los adultos mayores tenían puestos privilegiados, eran excelentes consejeros, jefes absolutos con poder legislativo y judicial (Polo y Martínez, 2001). De acuerdo con estos últimos autores, en aquella época Platón fue el principal defensor de la vejez y su concepción responde a una descripción ideal: la vejez es la culminación de la vida, libera de las pasiones juveniles y se disfruta de los placeres del espíritu. Promulgaba la gerontocracia: los ancianos deben gobernar y legislar.

En las sociedades tradicionales $y$ comunales los ancianos eran percibidos como autoridades naturales $y$ respetados por la mayoría de las personas y dado que representaban experiencia, conocimiento y sabiduría se les percibía como autoridades naturales (Polo y Martínez, 2001; Rodríguez, 1979). De tal manera que en la antigüedad, la concepción de la vejez era positiva, apoyada y respetada por la tradición oral y la costumbre; en esta los ancianos cumplían el papel de memoria colectiva, la experiencia denotaba prestigio, respeto, poder y sabiduría; además, la familia patriarcal jugaba una posición privilegiada. Sin embargo, con el desarrollo capitalista e industrial la concepción positiva de la vejez se desvanece. En palabras de Rodríguez (1979):

...la sociedad se urbaniza, tecnifica y dinamiza progresivamente; la experiencia deja de ser la principal fuente de conocimiento; las familias se dispersan, $y$ la producción se erige en valor dominante. En el marco de este nuevo sistema socioeconómico, a los ancianos se les asigna 
un papel marginal $y$ hasta se les cataloga de inútiles: ya no son productivos, han quedado 'obsoletos'. La vejez, que es un estadio natural de la persona, queda identificada con el 'retiro' o ‘jubilación' —que es una invención social- (p.78).

Lo anterior se acentúo con el neoliberalismo, donde la gran mayoría de los adultos mayores representan una carga económica derivada de su escasa capacidad para producir 0 en su caso, se les otorga una posición de no trabajadores, jubilados, omitiendo su importancia como personas. En contraste, la población en edades productivas, suele percibirse como sinónimo de competitividad y no adversidad al riesgo debido a la fuerza física y a la capacidad para incentivar la producción (Rodríguez, 1979).

Roberto Ham (1999) señala que la vejez representa importantes demandas de manutención y cuidado, con un claro retorno a la dependencia familiar en particular y hacia la sociedad en general. Tal deducción se encuentra cimentada en que el grupo poblacional de ancianos ha dejado de ser económicamente productivo, dependiente y son una carga debido a los cuidados y los servicios de salud que requieren, por tanto, la sociedad moderna ha trastocado las valoraciones positivas hacia las negativas, desplazando y desprotegiendo a los adultos mayores (Barreto, 2001).

Al respecto, Simone de Beauvoir (1970) critica la cultura elitista del capitalismo, la cual propicia la "deshumanización de la vejez", en la cual los viejos y los pobres son sinónimos; se describe la vida del anciano como vegetal y los últimos años de vida son considerados solo como un desecho y pérdida, prueba del fracaso de la civilización (Rodríguez, 1979).

En contraste con la postura "negativa" de la vejez, diversos Organismos Internacionales proponen una perspectiva alternativa mediante la denominación "Envejecimiento exitoso" (Baltes, 1997), "Envejecimiento funcional" (Gutiérrez, 1999) o "Vejez activa, productiva y saludable" (Naciones Unidas, 2002) con la finalidad de modificar la concepción de vejez como sinónimo de improductividad. El punto de convergencia enfatiza que la vejez y el éxito no son contradictorios (Castellano y De Miguel, 2010); por el contrario, las personas envejecidas deben gozar de oportunidades para seguir contribuyendo a la sociedad. Además, es preciso construir y consolidar sociedades intergeneracionales cimentadas en el mutuo respeto, la valoración recíproca y la equidad de género (Barreto, 2001; Naciones Unidas, 2002).

En síntesis, la dualidad de posiciones respecto a la vejez permite deducir que si bien, las condiciones de vida de un adulto mayor cambian radicalmente al llegar a un periodo de edad avanzada, esto no limita su pleno desarrollo. Sin embargo, el envejecimiento demográfico constituye un periodo de grandes retos para el Estado, la familia, la sociedad y los individuos.

\section{LA VEJEZ COMO UNA ETAPA HETEROGÉNEA: EL CASO DEL ESTADO DE MÉXICO}

La vejez, definida como los últimos años de vida, es una etapa distinta para los individuos (Ham, 1999) que no se puede calificar como un periodo homogéneo, sino en etapas que dependen de la edad y las condiciones sociales (Zetina, 1999). La diversidad de los grupos poblacionales envejecidos se asocia con el contexto social y las trayectorias de vida de cada individuo. Por ejemplo, en cuanto al estado de salud y capacidad, algunas personas adultas mayores poseen una condición de mayor dependencia con respecto a otras (Gutiérrez, 1999).

Dentro de esta heterogeneidad también se encuentran las condiciones sociales, económicas y el desarrollo de enfermedades (nutrición, estilos de vida y carencias en las primeras edades) que actúan desde edades tempranas y suelen profundizarse en la vejez (Gutiérrez, 1999; Moss, 2002; Pedrero, 1999). Lo anterior conduce, según Ham (1993), a distintas modalidades de obsolescencia, incapacidad y dependencia. Entonces, en la vejez se visualiza una gran heterogeneidad de situaciones (Reyes, 1999; Ham, 1999) vinculadas con elementos como la composición etaria, las condiciones de salud y económicas (CEPAL, 2006), así como del grupo social de pertenencia, del soporte o apoyo social, familiar y de género 
(Freixas, 1997; Rabell y Murillo, 2013; Rueda y Artazcoz, 2009).

Al respecto, Mercedes Pedrero (1999) ilustra la heterogeneidad de la población mayor de 60 años al enfatizar sobre las divergencias en la forma de vida, la edad de cada individuo, las diferencias de género, el lugar que ocupa en el hogar, la zona de residencia, así como, el grupo social al que pertenece. Particularmente, debe considerarse el género como parte de la vejez, pues las mujeres suelen vivir un número mayor de años que los hombres, lo que conduce a la 'feminización del envejecimiento' (Salgado de Snyder y Wong, 2007). En México, en 2010, la esperanza de vida en hombres fue de 73.1 años y 77.8 años en mujeres; se proyecta que en 2050 la esperanza de vida sea de 79.9 años para hombres y 83.9 años en mujeres (ConAPo, 2012).

A consecuencia de la no homogeneidad, Ham (1993) propone clasificar a los adultos mayores de acuerdo con rangos de edad y relacionarlos en concordancia al tipo de vejez que experimentan: a) periodo de transición hacia la vejez, si se encuentran entre los 60 a 64 años; b) vejez con oportunidades para una vida autónoma y buena salud, cuando están entre los 65 a 74 años, c) vejez avanzada con pérdida de capacidad $y$ dependencias crecientes para la salud, el sostenimiento y cuidado, de 75 a 84 años, $y$ d) periodo de decrepitud, dependencia total y preludio de la muerte, si las personas tienen más de 85 años.

En correspondencia con la diversidad de las trayectorias que puede seguir la población adulta mayor, la vejez puede ser analizada desde diferentes enfoques: demográfico, biológico, social, económico, familiar y psicológico (Ham, 1999). Debido al alcance de este artículo, únicamente se otorgará relevancia al enfoque social con la intención de identificar patrones de grupos entre el conjunto de adultos mayores a través de características individuales, familiares $y$ de contexto.

En términos poblacionales, el aumento de personas de 60 años es un fenómeno de alcance mundial. En México, según datos del Instituto Nacional de Estadística y Geografía (INEGI) vivían 6.9 millones de adultos mayores en el año 2000, cifra que se incrementó a 10.1 millones una década después (INEgi, 2000 y 2010). De acuerdo con los datos del año 2016, habitan en el país 12.5 millones, los cuales representan un $10,2 \%$. La expectativa es que para el 2030, este porcentaje aumente a $14,8 \%$ y en el año 2050 constituya $21,4 \%$ de la población nacional (conapo, 2012). Lo anterior implica que en un periodo de cinco décadas (2000-2050), la población adulta mayor mexicana se triplicará y con ello, sus necesidades.

El caso particular del Estado de México es sobresaliente debido a que es la entidad con el mayor número de personas envejecidas. Para el año 2010, la población mexiquense de 60 años o más ascendió a 1.1 millones, que representan $7,6 \%$ de la población estatal. Para el año 2015, fueron 1.5 millones de personas y representaron $8,9 \%$ del total de población de la entidad. Las proyecciones indican que para el año 2030 habrá en el Estado de México 2.9 millones de adultos mayores que constituirán $14,7 \%$ de la población total mexiquense. Estas cifras muestran que casi se duplica la cantidad de personas mayores de 60 años, lo que expone un ritmo de crecimiento acelerado de este grupo poblacional.

A partir de lo antepuesto, la estructura por edad y sexo de la población mexiquense de 60 años o más ostenta día con día una mayor presencia. El gráfico 1 muestra la composición por grupos quinquenales de edad y sexo de este segmento poblacional. 

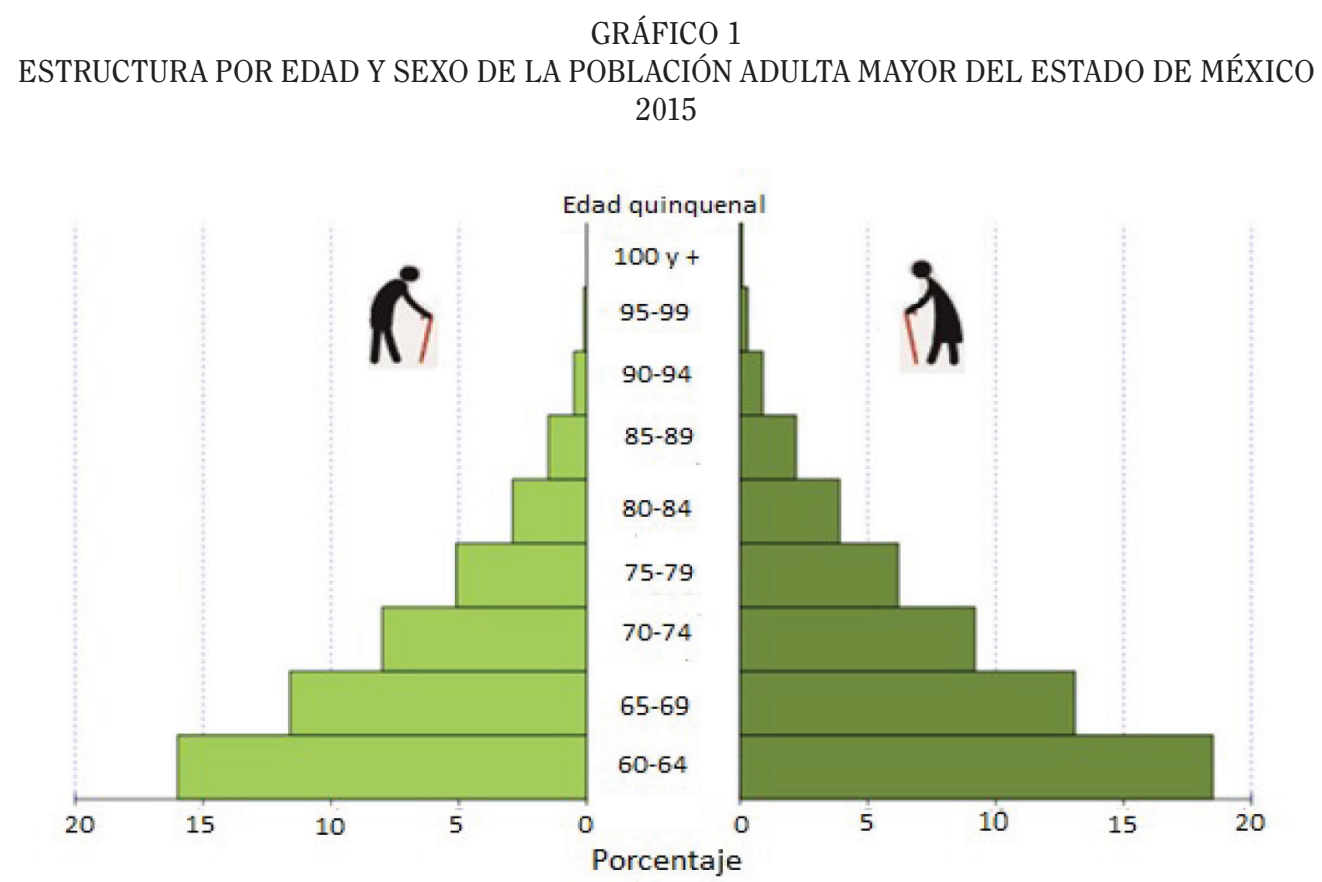

Fuente: $\quad$ Elaboración propia con base en la Encuesta Intercensal, 2015.

Como es notable, el gráfico 1 ilustra que la base está representada por el grupo quinquenal de 60 a 64 años, el cual registra la mayor proporción de adultos mayores (16\% corresponde a hombres y $18 \%$ a mujeres); a medida que aumenta la edad se observa una disminución de la participación de cada grupo quinquenal formando un triángulo. Igualmente, se puede apreciar una mayor proporción de mujeres adultas en contraste con los hombres de la misma edad para el conjunto de grupos quinquenales; por lo que corresponde un índice de masculinidad de 87 hombres por cada 100 mujeres en la población envejecida mexiquense.

Las distintas generaciones incluidas en la pirámide poblacional permiten identificar diversas trayectorias de vida, las cuales conducen a situaciones, problemáticas, contextos, clases y posiciones sociales que determinan grupos homogéneos y también segmentos radicalmente diferentes, palpados en adultos mayores bajo condiciones de pobreza en contraposición con adultos mayores cuyas condiciones de vida son aceptables.

En el apartado siguiente se ahonda acerca de la descripción de los indicadores y la estrategia metodológica llevada a cabo para alcanzar el objetivo planteado.

\section{FUENTE DE INFORMACIÓN Y METODOLOGÍA}

El proceso de envejecimiento poblacional puede ser analizado desde diferentes vertientes. En primer término, se encuentran las perspectivas relativas a aspectos biológicos y psicológicos; en segundo lugar, se hallan los enfoques relacionados con eventos culturales, sociales, económicos e incluso, demográficos. De la misma forma, este fenómeno puede ser examinado a partir de varios métodos complementarios que abarcan cuestiones cualitativas y cuantitativas. En particular, para indagar la vejez en la población mexiquense, esta investigación optó por retomar el enfoque social utilizando un método cuantitativo. Es importante 
señalar que este tipo de análisis tiene entre sus limitaciones mostrar solo una parte de las múltiples situaciones que enfrentan los adultos mayores mexiquenses.

Para este análisis se consideraron vertientes sociales que contienen doce variables que son la base para la conformación de grupos poblacionales de adultos mayores (Esquema 1).

Como se observa en el Esquema 1, las variables sociodemográficas que se consideraron fueron edad, sexo, nivel de escolaridad, tamaño de localidad y pertenencia indígena. Estas permiten mostrar diferencias sociodemográficas en la población envejecida del Estado de México. En la vertiente laboral se retomaron las siguientes variables: pensión o jubilación, condición de actividad y derechohabiencia. Los indicadores relacionados con estas permitieron indagar sobre la seguridad económica, la actividad de la población de 60 años o más y el acceso a servicios médicos, asimismo estas variables muestran el reconocimiento de derechos sociales y laborales.

VERTIENTES SOCIALES DE LA POBLACIÓN ADULTA MAYOR DEL ESTADO DE MÉXICO
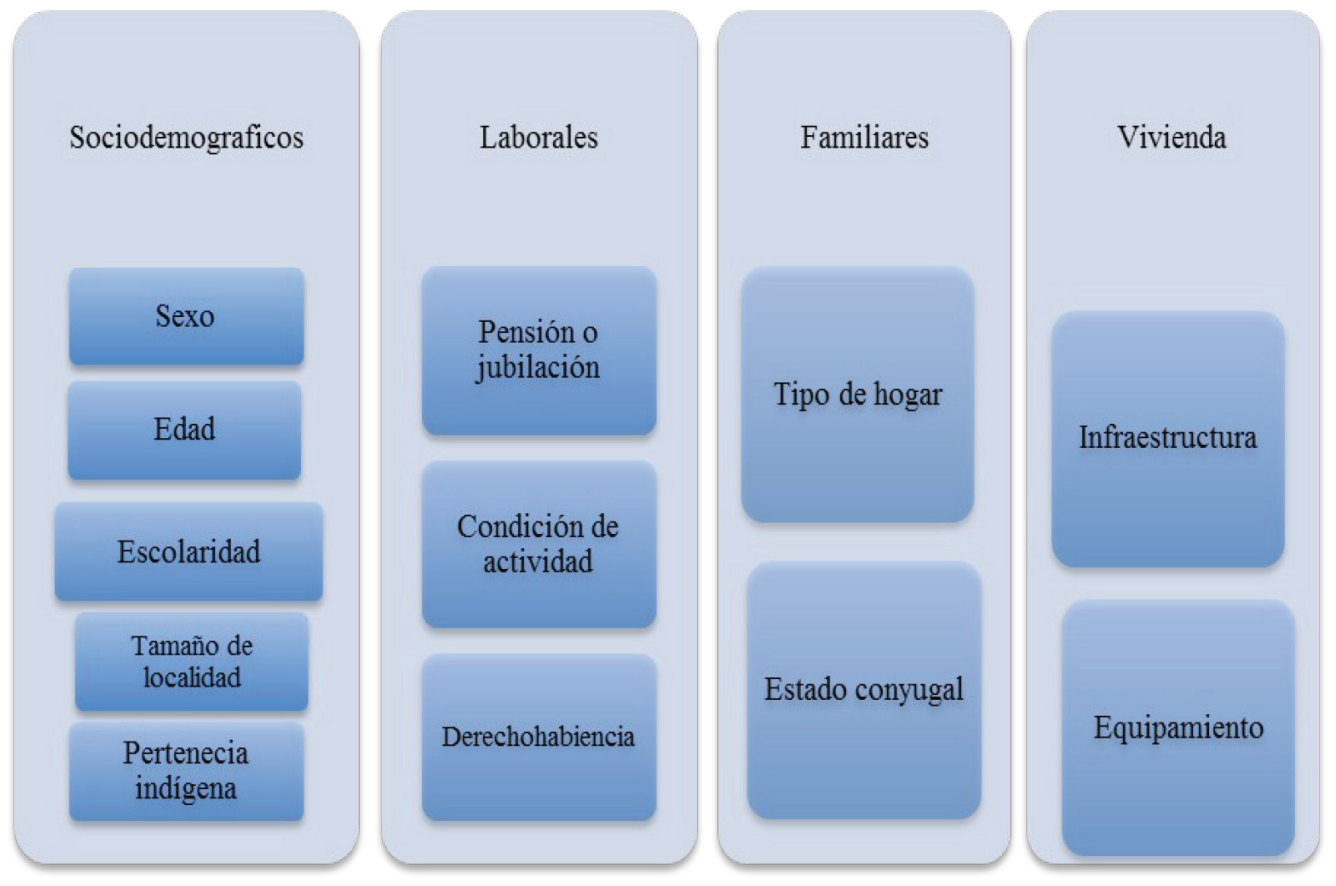

Fuente: Elaboración propia.

En lo que respecta al ámbito familiar se utilizaron los tipos de hogares que forman los adultos mayores y su estado conyugal. Estos indicadores son relevantes debido a que en la vejez, las redes familiares resultan fundamentales para mitigar las condiciones desfavorables de los adultos mayores y en muchos casos, la familia funge como un elemento de ayuda, soporte y asistencia (Solís, 1999; Villagómez y Sánchez, 2014).

Por último, en las características de la vivienda se hace referencia a la infraestructura 
$y$ el equipamiento con los que cuentan los hogares de la población de 60 años o más. El análisis del tipo de material de construcción de las viviendas de los adultos mayores, representa un elemento clave para determinar el nivel de riesgo al que está expuesta la población en estudio. Asimismo, se analiza el número de bienes que tienen en su vivienda, debido a que son considerados componentes esenciales del desarrollo, ingreso, salud, confort, durabilidad y confianza (Garrocho, 2013), incluso se ha reconocido su accesibilidad como parte de los derechos humanos (wно, 2006).

\section{FUENTE DE INFORMACIÓN}

Los indicadores se estimaron con datos de la Encuesta Intercensal 2015, cuyo objetivo es actualizar el volumen, la composición y la distribución de la población mexicana. Tal información permitió renovar los indicadores socioeconómicos, culturales y las condiciones de las viviendas de la población mexicana en el periodo intercensal (2015). El tamaño de muestra fue 6.1 millones de viviendas, con representatividad a nivel nacional, entidad federativa, municipal y para cada una de las localidades con 50 mil o más habitantes. La muestra de los adultos mayores mexiquenses ascendió a 171867 (InEgi, 2015). Se eligió la Encuesta Intercensal 2015 para desarrollar esta investigación, debido a que cuenta con las variables de estudio y es la base de datos más reciente que contiene la información para cumplir con el objetivo de esta investigación. No obstante, dentro de las limitaciones de esta fuente se encuentra la ausencia de información cualitativa que permita profundizar en las relaciones de apoyo social o comunitario de los adultos mayores.

\section{METODOLOGÍA}

Para esta investigación se estimó un Análisis de Correspondencias Múltiples (Асм) que es una técnica de reducción de datos que busca la asociación entre los valores de múltiples variables para mostrarla de manera gráfica en un mapa perceptual. Asimismo, es una herramienta de análisis de interdependencia que permite trabajar con variables no métricas y estudiar relaciones no lineales.

De tal manera que el Асм revela de forma gráfica el grado de asociación que tienen las variables; esquemáticamente los valores que tengan una relación fuerte aparecerán representados muy cercanos; mientras que aquellos que estén poco asociados se encontrarán más alejados. Así, con este método se podrán identificar perfiles sociales de la población de 60 años o más en el Estado de México tomando en cuenta las vertientes sociodemográfica, laboral, familiar $y$ de vivienda.

Uno de los requisitos para aplicar esta herramienta estadística es que las variables utilizadas en el estudio cumplan con los supuestos de ser mutuamente excluyentes y ser exhaustivas, según se muestra en el Cuadro 1. 
CUADRO 1

VARIABLES INCLUIDAS EN EL MODELO DE ANÁLISIS DE CORRESPONDENCIAS MÚLTIPLES

\begin{tabular}{|c|c|c|}
\hline DIMENSIÓN & VARIABLES & CATEGORÍAS \\
\hline \multirow{13}{*}{$\begin{array}{l}\text { Características } \\
\text { sociodemográficas }\end{array}$} & \multirow{2}{*}{ Edad } & 0: 60 a 69 años \\
\hline & & 1: 70 y más \\
\hline & \multirow{2}{*}{ Sexo } & 0 : Mujer \\
\hline & & 1: Hombre \\
\hline & \multirow{4}{*}{ Nivel de escolaridad } & 0 : Sin escolaridad \\
\hline & & 1: Básica \\
\hline & & 2: Medio superior \\
\hline & & 3: Superior y más \\
\hline & \multirow{2}{*}{ Pertenencia indígena } & $0:$ No \\
\hline & & $1: \mathrm{Si}$ \\
\hline & \multirow{3}{*}{ Tamaño de localidad } & 0 : Rural \\
\hline & & 1: Urbano \\
\hline & & 2: Metropolitano \\
\hline \multirow{7}{*}{ Aspectos laborales } & \multirow{5}{*}{ Condición de actividad } & $0:$ No trabaja \\
\hline & & 1: Trabaja \\
\hline & & 2: Jubilado \\
\hline & & 3: Hogar \\
\hline & & 4: Otra actividad \\
\hline & \multirow{2}{*}{ Servicios médicos } & 0 : Sin derechohabiencia \\
\hline & & 1: Con derechohabiencia \\
\hline \multirow{6}{*}{ Entorno familiar } & \multirow{3}{*}{ Tipo de hogar } & 0:Nuclear \\
\hline & & 1: Ampliado \\
\hline & & 2: Otro hogar \\
\hline & \multirow{3}{*}{ Estado conyugal } & 0 : Nunca unido \\
\hline & & 1: Alguna vez unido \\
\hline & & 3: Unido \\
\hline \multirow{4}{*}{ Características de la vivienda } & \multirow{2}{*}{ Infraestructura } & 0 : Infra. precaria \\
\hline & & 1: Infra. no precaria \\
\hline & \multirow{2}{*}{ Equipamiento } & 0: Un bien o menos \\
\hline & & 1: Dos o más bienes \\
\hline
\end{tabular}

Fuente: Elaboración propia. 
Las variables incluidas y el procedimiento metodológico propuesto permitieron identificar diferentes perfiles sociales vinculados a las características de los adultos mayores mexiquenses en el año 2015.

\section{CONTEXTO SOCIAL EN LA POBLACIÓN ADULTA} MAYOR DEL ESTADO DE MÉXICO EN EL AÑO 2015

Las vertientes sociales analizadas en esta investigación (sociodemográfica, laboral, familiar y de vivienda) representan elementos fundamentales para indagar el marco de desenvolvimiento en las condiciones de vida de los adultos mayores del Estado de México.

\section{CARACTERÍSTICAS SOCIODEMOGRÁFICAS}

En el año 2015, el Estado de México es la entidad con el mayor número de adultos mayores. De los 1.5 millones de personas de 60 años o más, $45,7 \%$ son hombres y $54,3 \%$ son mujeres, esta mayor presencia de población femenina se observa en todos los grupos quinquenales. La diferencia por sexo de los adultos mayores puede tener múltiples efectos en el ámbito social, familiar e individual, debido a que las necesidades de hombres y mujeres ancianos son relativamente diferentes en las esferas de la vida.

$\mathrm{Al}$ respecto, los estudios de género destacan que las mujeres presentan una mayor desventaja que los varones debido a que tradicionalmente estaban enfocadas a los quehaceres del hogar, así como, a la crianza y la educación de los hijos. Por lo anterior, las mujeres adultas mayores registran menores ingresos, estudios, empleo, jubilación, cobertura social. En contraste, las mujeres reciben mayor apoyo en redes sociales $y$ asistenciales para enfrentar los riesgos y cambios inesperados (Bueno y Rodríguez, 2008; Villagómez y Sánchez, 2014). Aunado a esto, es de considerar que las mujeres tienen una mayor esperanza de vida y con ello una mayor desventaja, ya que vivirán más tiempo para enfrentar una serie de retos que garanticen su supervivencia en sus últimos años de vida (Welti, 2013).

Para estas generaciones, el nivel de escolaridad es bajo, los resultados de la Encuesta
Intercensal 2015 muestran que el promedio de estudios alcanzado por las mujeres envejecidas del Estado de México es de 5.9 años, sexto de primaria, $y$ es incluso más bajo que el registrado por la población total (9.1 años). Además, el nivel de analfabetismo es alto, 4\% de los hombres no saben leer ni escribir un recado; proporción que se duplica para el caso de las mujeres $(10,5 \%)$. Este hecho coloca a las mujeres envejecidas en situación de desventaja y desigualdad, situándolas en una posición vulnerable.

Respecto a la diversidad cultural, los resultados de la Encuesta Intercensal 2015 muestran que $5,8 \%$ de los adultos mayores mexiquenses hablan alguna lengua indígena, donde predominan el mazahua y el otomí como las más importantes con $36,2 \%$ y $33,8 \%$, respectivamente. No obstante, $20 \%$ de toda la población adulta mayor se autoadscribe indígena, donde las mujeres tienen mayor presencia. La pertenencia indígena hace tener usos y costumbres propias de cada etnia, que constituyen elementos importantes para distinguir $y$ diferenciar a la población.

El tamaño de localidad de los adultos mayores mexiquenses muestra una concentración en áreas urbanas, es decir, con residencia en municipios metropolitanos o cercanos a la Ciudad de México. De acuerdo a la Encuesta Intercensal 2015, 61,8\% de los adultos mayores mexiquenses habita en lugares con alta densidad poblacional (más de 100 mil habitantes), $26,2 \%$ en localidades urbanas (de 2500 a menos de 100 mil habitantes) y 11,8\% en áreas rurales (menos de 2500 habitantes).

En este sentido, la ubicación de los adultos mayores según área de residencia hace que este grupo poblacional tenga diferentes trayectorias de vida. En las zonas urbanas al tener una mayor diversidad económica, laboral, social, de salud y de vivienda, así como mayor acceso a las vías de comunicación, hace que esta población pueda desempeñar una variedad de actividades económicas, mayor acceso a los servicios de salud, más bienes y servicios en las viviendas, factores importantes para disminuir el nivel de vulnerabilidad social. Mientras que en las zonas rurales hay menor apertura económica, mínimo acceso a empleos y limitadas vías 
de comunicación, aspectos que sugieren que los adultos mayores tienen un nivel de desprotección más elevado.

\section{ASPECTOS LABORALES}

Los resultados de la Encuesta Intercensal 2015 muestran que al menos uno de cada dos adultos mayores mexiquenses se encuentra desarrollando algún tipo de actividad económica. Las cifras muestran que 26,5\% trabajó mínimo una hora en la semana de referencia; mientras que un $34,8 \%$ se dedica a los quehaceres del hogar. En ambos casos sobresalen aquellos de 60 a 64 años como los de mayor participación.

Por su trayectoria laboral en el mercado de trabajo, aproximadamente uno de cada cinco adultos mayores son pensionados o jubilados, destaca el grupo de 60 a 69 años como el de mayor presencia; mientras que $5,2 \%$ tienen alguna limitación física o mental, siendo los de 70 a 79 años los que más se encuentran en esta condición y, como tal, en una situación de dependencia social, económica y familiar, pues requieren de asistencia y cuidados, algunos especiales.

Ahora bien, centrando la atención en aquellos adultos mayores que trabajan, los resultados permiten observar que estos tienen una mayor participación en actividades con condiciones laborales precarias que en empleos estables y bien remunerados. En este sentido, lo preocupante de las cifras de la Encuesta Intercensal hacen ver que existe una mayor participación de población adulta mayor en ocupaciones de menor calidad, menor productividad, menor ingreso y sin prestaciones laborales por estar relacionada más con actividades de cuenta propia $(49,8 \%)$ que como empleados u obreros (37,8\%), situación que los coloca como un grupo de población de mayor riesgo en términos económicos y sociales. Cabe destacar que en ambos casos sobresalen aquellos menores de 70 años de edad.

Lo anterior obliga a la población en estudio a depender de la familia en particular y de la sociedad en general, para poder cubrir sobre todo las demandas de manutención y cuidado que los coloca en situación de inseguridad familiar (Villagómez y Sánchez, 2014).
Respecto a la cobertura o el derecho que tienen los adultos mayores de recibir atención médica, la información estadística muestra que $44,7 \%$ no tienen derecho a servicios médicos, siendo los más vulnerables aquellos mayores de 80 años; lo anterior podría deberse a que desarrollan actividades por cuenta propia.

Del total de la población adulta mayor del Estado de México, 40\% tienen derecho a recibir atención médica en el Instituto Mexicano del Seguro Social (IMSS), 7,2\% en el Instituto de Seguridad y Servicios Sociales de los Trabajadores del Estado (ISSSTE), 2,3\% en el Instituto de Seguridad y Servicios Sociales de los Trabajadores del Estado a nivel Estatal (ISSSTE estatal), 2,4\% en un seguro privado y 1,1\% en Pemex, Defensa o Marina, siendo en todos los casos los adultos de 60 a 69 años de edad los que tienen más presencia en este beneficio; este derecho proviene ya sea por haber trabajado como asalariado o por continuar trabajando ${ }^{4}$.

Así, las cifras anteriores reflejan una situación de desprotección social para un parte importante de adultos mayores, debido a que no toda la población puede asegurar su bienestar a través del acceso a los servicios médicos y que están expuestos a una gran cantidad de situaciones perjudiciales que afectan su salud física $y$ mental; de la misma forma no cuentan con todas las prestaciones que ayudan a mejorar el nivel de vida.

\section{ENTORNO FAMILIAR}

En lo que respecta al ámbito familiar, en el año 2015 según la Encuesta Intercensal, alrededor de $87,9 \%$ de los adultos mayores viven con otro familiar. Específicamente, 44,7\% forma parte de una familia ampliada; mientras que 43,2\% tienen un hogar nuclear.

4 Para esta investigación no se consideraron a las personas que cuentan con seguro popular por dos razones: en primer lugar, el seguro popular es un programa gubernamental de asistencia social por lo que no se otorga por derechohabiencia. En segundo lugar, la calidad de servicios en las instituciones es diferentes; de ahí que contar con seguro popular no necesariamente cubre todas las necesidades de salud que puedan tener los adultos mayores. 
Una situación de desventaja grave se podría presentar cuando el adulto mayor vive solo; en esta situación están casi 140000 mexiquenses. Esta condición puede manifestar rasgos de abandono, depresión o aislamiento. Además, incrementa el riesgo de que ocurra algún accidente y no se tenga ayuda cercana.

Respecto a la conformación familiar, los resultados permiten visualizar dos aspectos: el primero que una importante proporción de población de 60 años o más aún tiene la capacidad, la fuerza y el respeto para dirigir un hogar (61,1\%); y el segundo, las marcadas diferencias de género, en las cuales los hombres casi en su totalidad son jefes de hogar y las mujeres son compañeras o esposas.

El estado civil es otra de las variables que se analizan del entorno familiar, los resultados muestran que en la entidad mexiquense alrededor de $61,8 \%$ viven en pareja, casados o en unión libre; $33,1 \%$ estuvieron alguna vez unidos, de los cuales $26,2 \%$ se encuentra en situación de viudez y 6,9\% separados o divorciados; mientras que $4,7 \%$ nunca se unieron. La información anterior expone una diversidad de situaciones familiares de los adultos mayores que acentúan las diferencias entre esta población.

\section{CARACTERÍSTICAS DE LAS VIVIENDAS}

En las características de la vivienda es importante destacar las adversidades que enfrenta la población adulta mayor del Estado de México. Según los datos, alrededor de 7\% de los ancianos vive entre paredes con materiales poco seguros $y$ duraderos, $13,5 \%$ tiene techos poco favorables y $5,9 \%$ carece de un espacio para cocinar y vive en una sola habitación.

La situación anterior coloca a este grupo poblacional en situación de riesgo, tanto a ellos como a todos los integrantes del hogar, ya que las precarias condiciones de la vivienda impactan directamente en su salud y el nivel de vida.
Asimismo, la información estadística evidencia que una proporción considerable de adultos mayores del Estado de México carece de ciertos bienes en la vivienda. Los casos más preocupantes de la Encuesta Intercensal muestran que $40 \%$ no tiene refrigerador en su hogar, 5,5\% cuenta con fogón de chimenea, $66,2 \%$ no cuenta con lavadora en casa. Estas carencias pueden generar enfermedades, deteriorar su calidad de vida $y$ en general, exponerlos a un mayor número de riesgos dentro de la vivienda.

El contexto social expuesto en líneas precedentes muestra la heterogeneidad que experimenta la población adulta mayor del Estado de México. En la sección subsecuente se identifican los perfiles sociales de los adultos mayores mexiquenses mediante el método de Análisis de Correspondencias Múltiple.

PERFILES SOCIALES DE LAS PERSONAS DE 60 AÑOS O MÁS EN EL ESTADO DE MÉXICO EN EL AÑO 2015

La información presentada anteriormente expone que el proceso de envejecimiento en el Estado de México es complejo y heterogéneo; sin embargo, a partir de la aplicación del Análisis de Correspondencias Múltiples (Асм) es posible identificar cinco grupos de adultos mayores. Los resultados del Асм permiten observar que existe consistencia entre las doce variables incluidas en el modelo, debido a que agrupan a la población adulta mayor en categorías con características similares y al mismo tiempo, con diferencias entre los grupos.

En el gráfico 2 es posible identificar a los cinco grupo de adultos mayores que se distribuyen en los cuatro cuadrantes, en especial en el cuadrante i se identifica a los adultos mayores que presentan un nivel social alto; de lado opuesto, cuadrante iII, se ubica a la población adulta mayor en un nivel social muy bajo. 


\section{GRÁFICO 2 \\ ANÁLISIS DE CORRESPONDENCIAS MÚLTIPLES}

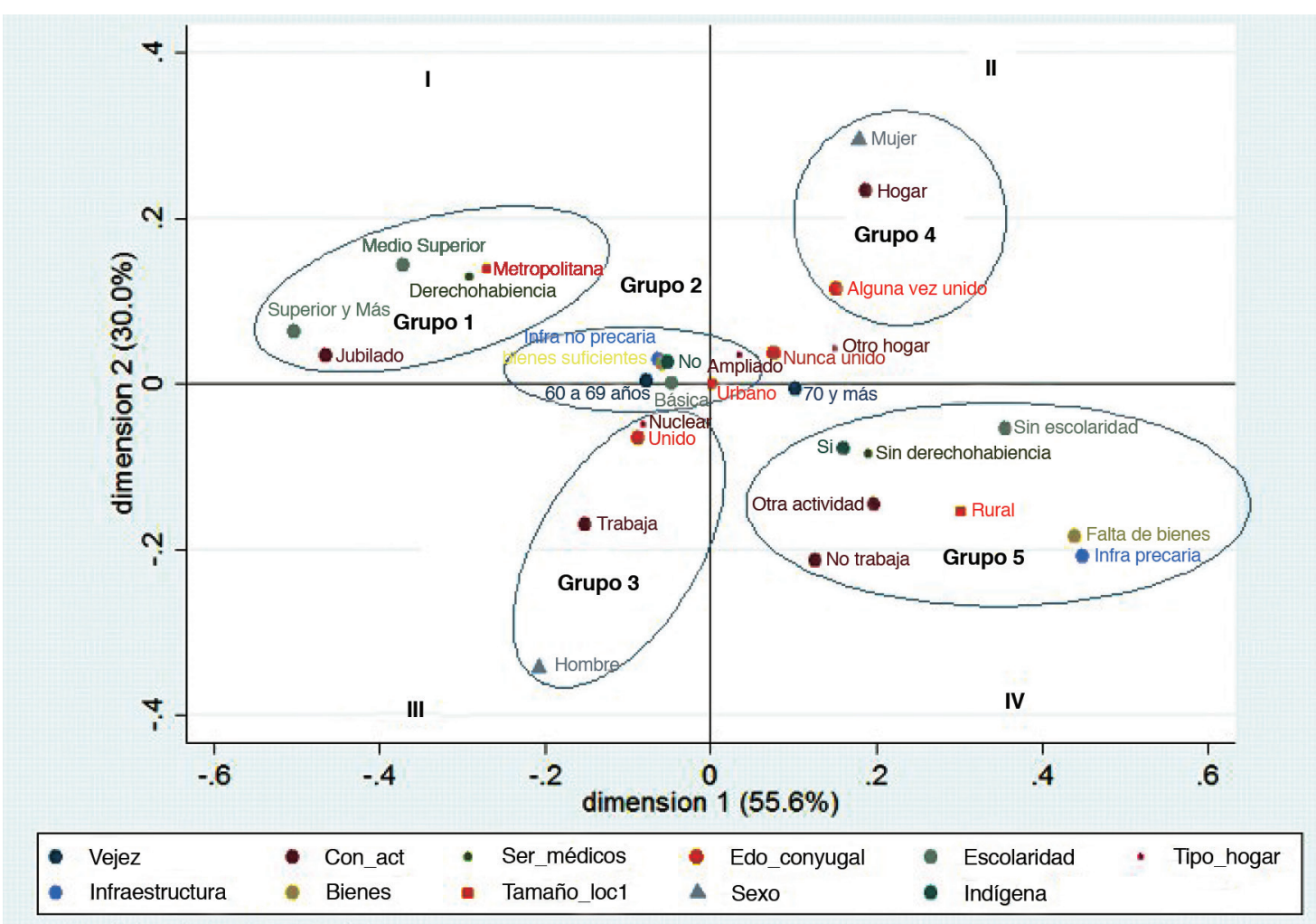

coordinates in principal normalization

Fuente: Elaboración propia con base en la Encuesta Intercensal, 2015.

A continuación se describen los cinco grupos sociales de adultos mayores identificados a partir de vertientes sociales (sociodemográfica, laboral, familiar y de vivienda).

a) Grupo 1. Nivel alto: (ubicado en el cuadrante I con valores negativos en el primer eje y positivos en el segundo), este grupo se forma por adultos mayores que tienen protección social amplia. Por un lado, tienen acceso a los servicios de salud y por otro, reciben una pensión o jubilación; residen principalmente en alguna de las dos zonas metropolitanas de la entidad mexiquense - Toluca o México- o en municipios con más de
100 mil habitantes. Otra característica que comparte este grupo es el alto nivel educativo -medio superior, superior o posgrado (lo que equivale a 12 años o más de escolaridad)—, que para dichas generaciones se considera elevado, si se toma como referencia que el promedio nacional de escolaridad es de 9.1 años, que equivale a tercero de secundaria.

b) Grupo 2. Nivel medio: (ubicado en el centro del gráfico 2 con una tendencia hacia el cuadrante I), este grupo está formado por adultos mayores que acaban de entrar a la senectud o son menores de 70 años, es decir, se encuentran en la etapa 
umbral del envejecimiento; además, son parte de familias ampliadas con la posibilidad de completar el ingreso del hogar con varios integrantes; sus viviendas son aceptables y están bien equipadas, lo que implica más comodidad y mejores condiciones de convivencia; residen principalmente en zonas urbanas -entre 2500 $y$ menos de 100000 habitantes-; su estancia en las ciudades implica poca asociación a pertenecer a alguna etnia indígena. El nivel de escolaridad alcanzado por este grupo es de educación básica, alrededor de 9 años, similar al registrado a nivel nacional.

c) Grupo 3. Nivel medio bajo: (localizado en el cuadrante iv con valores negativos en ambos ejes), en este grupo se sitúa a los hombres adultos mayores con nexos en el mercado laboral porque aún trabajan, están unidos y forman hogares nucleares. La condición de ocupados como actividad importante de este grupo sugiere diversos aspectos, entre los que sobresalen: por un lado, la necesidad de continuar en el ámbito laboral para obtener ingresos y satisfacer las condiciones de vida, situación que conduce a prolongar la jubilación y por otro lado, la inexistencia de esta prestación laboral - jubilación- lleva a que permanezcan ocupados mientras la salud se los permita. Como tercer punto, la decisión propia de permanecer ocupados por las buenas condiciones físicas y mentales de los adultos mayores.

d) Grupo 4. Nivel bajo: (ubicado en el cuadrante il con valores positivos en ambos ejes), en este grupo se encuentran las mujeres adultas mayores que se dedican a los quehaceres del hogar $y$ que alguna vez estuvieron unidas. Este conjunto representaría a un sector vulnerable por dos razones: la primera porque las mujeres de estas generaciones tuvieron poca presencia en el mercado laboral, lo que se relaciona con una desprotección social formal que podría estar vinculada con dedicarse a las actividades exclusivas del hogar, así como a las actividades de cuidado.

La segunda razón es por el estado de civil, la viudez es la situación conyugal más común cuando las mujeres adultas alguna vez estuvieron unidas; en este sentido, la viudez coloca en circunstancias de desventaja $y$ vulnerabilidad a la población femenina al no contar con una pareja para el apoyo económico, social, psicológico y de compañía.

e) Grupo 5. Nivel muy bajo: (se localiza en el cuadrante III con valores positivos en el primer eje $y$ negativos en el segundo), en este grupo se encuentran los adultos mayores que viven en el contexto rural localidades con menos de 2500 habitantes-, que tienen viviendas precarias $y / 0$ pocos bienes en el hogar, que no desarrollan alguna actividad económica remunerada y con escasa protección social. Además, este conjunto de adultos mayores tiene una presencia importante en alguna etnia indígena; de las once lenguas en la entidad mexiquense, el grupo mazahua y el otomí son los dos más importantes. Respecto al nivel de escolaridad, este grupo es el de mayor desventaja social debido a que se registra nulo grado escolar; variable fundamental para la desigualdad laboral, económica y de salud.

En el perfil social de los cinco grupos, anteriormente mencionados, es posible identificar un aspecto fundamental que se asocia con el sexo, el nivel de escolaridad y la jubilación, ya que estas variables agudizan la desigualdad entre los adultos mayores. En el caso del sexo sobresalen las diferencias de género, debido a que las mujeres y los hombres adultos mayores se ubican en lados opuestos (Gráfico 2), esto implica importantes distancias sociales entre hombres y mujeres. En el nivel de escolaridad se identifica una marcada desigualdad entre los adultos mayores asociados al tamaño de la localidad: sin escolaridad (ámbito rural), con nivel 
básico (ámbito urbano) y nivel medio superior, superior o posgrado (metropolitano). Asimismo, la jubilación o pensión colocó a los adultos mayores con el nivel social más alto, marcando así la desigualdad social entre los grupos.

Es importante señalar que el hecho de agrupar a la población adulta mayor bajo ciertas particularidades no significa necesariamente que el conjunto de los adultos compartan todas las características o se aplique como regla general, sino que se trata de asociaciones que permiten establecer rasgos poblacionales en cada uno de los grupos.

El Análisis de Correspondencias Múltiples confirma las distintas trayectorias seguidas por los adultos mayores, que dan pie a diversas situaciones, problemáticas y contextos. Para Rodríguez (1979), en el grupo de los adultos mayores se reproducen las diferencias estructurales y estratificaciones de la sociedad en la que se encuentran insertos: según sea su clase y posición social. Lo anterior conduce, según el autor, a que los ancianos perciban diferentes ingresos, vivan en distintos lugares y tengan acceso a diversas oportunidades $y$ actividades. Según Ham (2014) "en todas partes la heterogeneidad de la vejez no es solo en función de la edad, sino que depende de la posición social y económica" (p.25).

Asimismo, la evidencia empírica apoya lo que Ham (1993) y Pedrero (1999) mencionan respecto a la importancia que tiene la edad de la persona en los aspectos sociales y económicos. Al respecto, se puede observar que aquellos individuos de 60 a 69 años, como lo afirma Ham (1993), están en transición hacia la vejez con oportunidades aún laborales, autosuficientes y con buena salud, colocándose en buenas condiciones socioeconómicas. Mientras que aquellos ancianos mayores de 70 años, ya se encuentran en vejez avanzada, con dependencias crecientes para la salud, por lo tanto, con malas condiciones sociales y económicas.

El presente análisis muestra que el género, aproximado a través de la variable sexo, se asocia con el nivel social; por tanto, hombres y mujeres adultos mayores tienen características sociales diferentes.
Por otro lado, el nivel de escolaridad resalta como variable que influye en el nivel de vida de esta población. En esa misma línea, la condición de jubilado o pensionado también resulta un aspecto determinante para los adultos mayores, debido a que los jubilados son las personas que registran el nivel más alto, de ahí la importancia que tiene este derecho laboral en la población de 60 años o más. En este sentido, Pedrero (1999) menciona que "la única posibilidad que tiene un viejo de sobrevivir dignamente es recibiendo la retribución justa por su contribución laboral en el pasado, esto es, a través de una pensión de retiro justa" (p.80).

\section{CONCLUSIONES}

Los resultados obtenidos respecto al perfil social de los adultos mayores mexiquenses permiten establecer ciertas conclusiones sobre los efectos del sexo, el nivel educativo y la jubilación en el envejecimiento.

Concretamente, el sexo es un factor que produce y reproduce desigualdades de género, que se presentan desde tempranas edades hasta las últimas etapas de la vida. En lo que respecta a la escolaridad se asocia con una elevada vulnerabilidad, debido a que impacta en la trayectoria laboral de la población; mientras que la variable de jubilación se relaciona con la desigualdad económica que repercute en el nivel de vida de las personas envejecidas mexiquenses.

Con base en lo anterior, los adultos mayores del Estado de México presentan múltiples desigualdades que agudizan las problemáticas sociales que tiene este grupo de población.

De esta manera, los resultados de la investigación presentan dos aspectos centrales de los perfiles sociales de la población estudiada en el año 2015. Por un lado, la heterogeneidad de la población envejecida del Estado de México reflejada en la diversidad de grupos. Por el otro, la desigualdad entre perfiles sociales que se manifiesta en las distancias del Асм.

En este sentido, los adultos mayores del Estado de México en el año 2015 presentan una heterogeneidad vertical que refleja importantes diferencias que complejizan las interacciones sociales de este grupo poblacional. Lo anterior expone, que en el actual modelo 
neoliberal, la desigualdad atraviesa todas las etapas de la vida y que en los adultos mayores, esta característica social no es la excepción.

Por último, una de las líneas de investigación que se podría generar a partir de este trabajo versa sobre las relaciones entre cada una de las interacciones sociales que se presentan alrededor de la población envejecida, como es el caso de la desigualdad de género o el acceso a los sistemas de jubilación. La finalidad de realizar los análisis propuestos estaría relacionada con el diseño de políticas públicas integrales enfocadas a la atención de la heterogeneidad entre perfiles sociales de los adultos mayores mexiquenses.

\section{REFERENCIAS}

Arber, S. y Ginn, J. (1995). Mera conexión, relaciones de género $y$ envejecimiento. Relación entre género y envejecimiento: un enfoque sociológico. Madrid, España: Narcea.

Arzate-Salgado, J., Fuentes-Reyes, G., y RetelTorres, C. (2007). Desigualdad y vulnerabilidad en el colectivo de Adultos mayores en México y en el Estado de México: Una revisión multidisciplinaria. Quivera, 9 (2), 231-262.

Baltes, P.B. (1997). Emergence of a powerful connection between sensory and cognitive functions across the adult life span: A new window to the study of cognitive aging? Psychology and Aging, 12 (1), pp: 12-21.

Barreto, L. (2001). Maltrato y exclusión en las personas mayores. Congreso Internacional sobre Maltrato al Adulto Mayor "una realidad oculta", Lima, Perú.

Barrientos, A. (2006). Ageing, poverty, and public policy in developing countries: New Survey Evidence. Institute of Development Studies at the University of Essex.

Beauvoir, S. (1970). La vejez. Buenos Aires, Argentina: Sudamericana.

Bourdieu, P. (2005). Capital cultural, escuela y espacio social. México: Siglo xxı.

Bueno, E. y Rodríguez, G. (2008). Una aproximación a la vulnerabilidad por género. Los referentes del empleo y la pobreza. ALAP: pobreza y vulnerabilidad social. Enfoques y perspectivas. Serie Investigaciones 3, Asociación Latinoamericana de Población, Río de Janeiro, Brasil.

Castel, R. (2006). Inseguridad social ¿Qué significa estar protegido? Buenos Aires, Argentina: El Manantial,

Castellano, C. y De Miguel, A. (2010). Estereotipos viejistas en ancianos: actualización de la estructura factorial y propiedades psicométricas de dos cuestionarios pioneros, International Journal of Psychology and Psychological Therapy, 10, (2), 259-278.

Comisión Económica para América Latina y el Caribe (cEPaL). (2006). Demografía del envejecimiento. Capítulo 1. Manual sobre indicadores de calidad de vida en la vejez, Santiago de Chile. Consultado el 23 de febrero de 2016, recuperado de http:// www.bvsde.paho.org/bvsacd/cd65/vejez/ cap1.pdf

Consejo nacional de Población (conapo). (2012). Proyecciones de Población y Vivienda, 2000-2050. México, D.F. Consultada 5 de abril de 2016, recuperado de http:// www.conapo.gob.mx/es/CONAPO/ Proyecciones_Datos

Freixas, A. (1997). Envejecimiento y género: otras perspectivas necesarias. Anuario de Psiocología, 73, 31-42.

Garrocho, C. (2013). Dinámica de las ciudades de México en el siglo хxı. Cinco vectores clave para el desarrollo sostenible. Zinacantepec, México: unfpa, conapo, El Colegio Mexiquense.

Gutiérrez, L. M. (1999). El proceso de envejecimiento humano: algunas implicaciones asistenciales y para la prevención. Papeles de Población, 5, (19), 125-147.

Ham, R. (1993). México: país en proceso de envejecimiento. Comercio Exterior, Banco Nacional de Comercio Exterior, $43(7)$.

Ham, R. (2014). El envejecimiento en México: el siguiente reto de la Transición demográfica. Primera edición. Tijuana, Baja 
California, México: Miguel ángel Porrúa, El Colegio de la Frontera Norte.

Ham, R. (enero-marzo, 1999). El envejecimiento en México: de los conceptos a las necesidades. Papeles de Población, 5 (19), 7-21.

Hernández, M. (2000). Desigualdades según género en la vejez. Barcelona: Secretaría Sectorial de la Mujer y de la Juventud. Recuperado de https://igualdadyviolenciadegenero.carm.es/documents/202699/216982/Desigualdades+s eg\%C3\%BAn+g\%C3\%A9nero+en+la+v ejez/fa74002d-0b2f-4abb-b215-1405ee$8 \mathrm{~d} 4 \mathrm{f} 35$

Instituto Nacional de Estadística y Geográfica (Inegi). (2000). Censo de Población y Vivienda 2000. Aguascalientes, México. Consultado el 2 de febrero de 2016, recuperado de http://www.INEGI.org.mx/est/ contenidos/proyectos/ccpv/default.aspx

Instituto Nacional de Estadística y Geográfica (InEgi). (2010). Censo de Población y Vivienda 2010. Aguascalientes, México. Consultado el 20 de abril de 2016, recuperado de http://www.INEGI.org.mx/est/ contenidos/proyectos/ccpv/default.aspx

Instituto Nacional de Estadística y Geográfica (INEGI). (2011). Principales Comparaciones Internacionales del PBI. Consultado el 20 de mayo de 2016, recuperado de http:// www.INEGI.org.mx/est/contenidos/proyectos/pci/mapa_pci/mapa.html

Instituto Nacional de Estadística y Geográfica (INEGI). (2014). Aportación al Producto Interno Bruto (PBI) nacional. Consultado el 15 de enero 2016, recuperado de http://cuentame.INEGI.org.mx/monografias/informacion/mex/economia/pib. aspx?tema

Instituto Nacional de Estadística y Geográfica (InEgi). (2015). Encuesta Intercensal, 2015. Microdatos. Consultado el 3 de marzo de 2016, recuperado de http:// www.INEGI.org.mx/est/contenidos/proyectos/ccpv/default.aspx

Instituto Nacional de Estadística y Geográfica (Inegi). (2016). Comparaciones Internacionales. Recuperado de http:// www.beta.INEGI.org.mx/temas/pci/
Juárez, C., Márquez, M., Salgado, N., Pelcastre, B., Ruelas, M. y Reyes, H. (2014). La desigualdad en salud de grupos vulnerables de México: adultos mayores, indígenas y migrantes. Revista Panamericana de Salud Pública, 35(4), 284-290.

Leñero, L. (enero-marzo, 1999). Implicaciones intrafamiliares de la población en la tercera edad. Papeles de Población, 5 (19), 199-215.

Moss, N. (2002). Gender equity and socioeconomic inequlity: a framework for the patterning of women's health. Social Science \& Medicine, 54, 649-661.

Organización de las Naciones Unidas. (2002). Segunda Asamblea Mundial sobre Envejecimiento. Madrid, España.

Pedrero, M. (1999). Situación económica en la tercera edad. Papeles de Población, 5 (19), 77-101.

Polo, M. y Martínez, M. (2001). Visión histórica del concepto de vejez en las sociedades antiguas. Cultura de los Cuidados, V (10), 15-20.

Rabell, C., y Murillo, S. (2013). Apoyos recibidos por personas de la tercera edad en México, en Montes de Oca, V. (coord.), Envejecimiento en América Latina y el Caribe. México: unam.

Redondo, N. (1990). Ancianidad y Pobreza: una investigación en sectores populares urbanos. Buenos Aires, Argentina: Humanitas.

Reyes, L. (enero-marzo, 1999). La vejez indígena. El caso de los zoques del noroeste chiapaneco. Papeles de Población, 5 (19), 173-197.

Rodríguez, J. (1979). Perspectiva sociológica de la vejez. Revista Española de Investigaciones Sociológicas (7), 78-97.

Rueda, S. y Artazcoz, L. (2009). Gender inequlity in health among elderly people in a combined framework of socioeconomic position, family, characteristics and social support. Ageing \& Society (29), 625-647.

Salgado de Snyder, N. y Wong, R. (2007). Género y pobreza: determinantes de la salud en la vejez. Salud Pública de México, 49 (4), S515-S521. 
Secretaría de Desarrollo Social (2013). Diagnóstico del Programa de Pensión para Adultos Mayores. México: Secretaría de Desarrollo Social. Recuperado de http://www.gob.mx/sedesol/documentos/ diagnosticos-de-los-programas-de-desarrollo-social

Solís, P. (1999). El ingreso a la cuarta edad en México: una aproximación a su intensidad, calendario e implicaciones en el apoyo familiar y social a los ancianos. Papeles de Población, vol. 5 (19), 43-63.

Treviño-Siller, S., Pelcastre-Villafuerte, B. y Márquez-Serrano, M. (2006). Experiencias de envejecimiento en el México rural. Salud Pública de México, 48 (1), 30-38.

Van de Poel, E., Reza, A., Speybroeck, N., Van Ourti, T. y Vega, J. (2008). Socieonomic inequality in malnutrition in developing countries. Bulletin of the World Health Organization, 86 (4), 282-291.

Villagómez, G. y Sánchez, M. (2014). Mujeres mayas: envejecimiento, pobreza y vulnerabilidad. Península, IX (2), 75-98.

Welti, C. (2013). Política social y envejecimiento. Papeles de Población,(77), 25-59.

World Health Organization's (wно). (2006). Meeting the $M D G$ drinking-water and sanitation target. The urban and rural challenge of the decade. Ginebra, Suiza.

Zetina, M. G. (1999). Conceptualización del proceso de envejecimiento. Papeles de Población, 5 (19),23-42.

Fecha de ingreso: 04/11/2016

Fecha de aprobación: 28/08/2017 